\title{
Research on Salary System Management of Hospital union
}

\author{
Guanzhuo Wang, Daxu Liu, Jiana Wang, Yang Mu \\ Heilongjiang University of Chinese Medicine, Jiamusi, Heilongjiang, 154002
}

Keywords: Salary Management, Hospital union, Medical Development.

\begin{abstract}
The construction of regional Hospital union is an important part of deepening the reform of medical and health system. It is an important way to promote the construction of regional Hospital union and enhance the level of primary health care service. Through the comparison of medical and health expenditure and doctor 's income level in some countries, this paper analyzes the shortcomings of the salary system of public hospitals in China and points out the direction of salary system reform. Results: The relative shortage of domestic medical and health expenditure, the doctor's salary level and its labor pay does not match the salary system is relatively unreasonable, ignoring the role of internal remuneration. China must increase the financial investment in the hospital, improve the level of pay, establish a reasonable performance indicator, improves the internal compensation system.
\end{abstract}

\section{Introduction}

Regional medical consortium is a medical resource in the same area, usually by a regional tertiary hospital and secondary hospital, community hospital, village health room composed of a Hospital union, referred to as Hospital union. By constructing a new type of medical and health service system based on vertical Hospital union, it is not only helpful to promote the further optimization of the layout of medical resources, but also has positive significance to continuously promote the quality of medical resources. The so-called subsidence is the quality of medical resources from the city hospitals, urban district medical centers, subsided to secondary hospitals and community health service centers, township hospitals and other primary health care institutions, making the layout of high-quality medical resources more reasonable, more configuration science. Through the link between the Hospital union and the bridge, it will help the city public hospital and the grassroots medical and health institutions to establish a better linkage between the upper and lower linkage, the division of labor operation mechanism, so that the regional medical human resources vertical flow further smooth, primary medical service capacity And further improve the overall performance of the medical and health service system to further improve, so as to more effectively promote the implementation of hierarchical treatment system for the city and the grassroots people to provide a more secure, effective, convenient, cheap, continuous, high quality basic medical and health services.

\section{Definition and Purpose of "Hospital Union"}

"Hospital union" is the "Hospital union" short. For what is the "Hospital union", there is no uniform definition. Different scholars in the exploration of a different point of view: Sun Xizi and so that "Hospital union" refers to a certain area of medical resources integrated together by a tertiary hospital joint a number of secondary hospitals and primary health care Institutional composition ", emphasizing the" Hospital union "should be part. Zhenggang that the "Hospital union" refers to a certain area by different types, levels of medical and health institutions organic combination, the formation of cooperative alliances or medical groups, as the community of interests and the community of responsibility, "stressed that the" Hospital union "should be on the residents Health 
of the whole process is responsible for the high. Hao Xichun that, because the different levels of medical institutions, there are differences in asset properties, breakthrough property rights obstacles are difficult, so that " Hospital union" is not breaking the original ownership relationship, three Level hospitals, community hospitals and community health service centers to strengthen the joint, so that medical resources can be shared in the Commonwealth. "Lin handle that" Hospital union "should be led by the top three hospitals, specialist hospitals to participate in a wide range of uniform distribution of the community, And specifically refers to the establishment of a consortium in the region, emphasizing the concept of "Hospital union" and "region", and pointed out that "Hospital union" should be called "regional wisdom health service system", and even CDC Can be included in the system, the system not only provides medical services, but also the health management of the above-mentioned scholars point of view, from the "Hospital union" of the relevant policy This study suggests that the specific definition of "Hospital union" is dynamically changing with the process of its continuous development and evolution. For the time being, "Hospital union" should be composed of different levels of medical institutions to Medical resources integration for the purpose of providing health services with the unity of residents health management.

China's health resources allocation is unreasonable, high-quality resources concentrated in the city, subordinate medical institutions, especially below the county level of medical institutions lack of health resources, and the needs of the masses do not match, coupled with the general practitioner training speed, level and ability Poor, leading to the grassroots level is difficult to become the first diagnosis of the task, making it difficult to truly implement the classification of diagnosis and treatment. Patients regardless of the priorities of the disease, are concentrated flock to the big hospitals, leading to a large hospital on the 1st hard to find, and most communities and township hospitals can be spared, resource constraints waste. Quality resources sink difficult, resulting in spontaneous transfer to the higher medical institutions, resulting in confusion in the treatment order. If you want to attract patients to the grassroots level, we must first high-quality resources with the grassroots. And "Hospital union" is a worth exploring the "strong grass-roots" approach. Undeniably, now a lot of large hospitals held "Hospital union" is a very important purpose is to seize the market. On the one hand, through the multi-built contact points, referral and other means to obtain benefits, on the other hand, with the expansion of the scale of the Commonwealth, siphon can be more human resources and the source of knowledge to the core homes. However, the "Hospital union" the real purpose should be like a pipeline, the large hospitals and grass-roots medical institutions linked to achieve high-quality resources sink.

\section{The Current Hospital Union Salary Management Malpractice}

Our Hospital union is a public institution, and the remuneration system is limited to the institution system. The basic salary of doctors is in accordance with the 2006 income system of income distribution system reform program, according to the staff positions and different levels, the implementation of national unified policies and standards, the implementation of job performance wage system. The salary is composed of basic salary, performance pay, subsidy, basic salary including salary, salary, performance pay and basic performance pay and incentive performance pay, but there are studies that show the basic role of the incentive Wages less than $30 \%$. Compared with some countries, China's health care expenditure ratio of GDP is too low.

At present, the heavy workload of our doctors and high-tech level is not reflected in the remuneration and social status. It is a common phenomenon for doctors to be paid in the world. According to the study found that the doctor's wages are generally higher than the social average wage of 2.5-4.0 times, but the average salary of our Hospital union doctors only 6000 yuan, only the social average wage of about 1.4 times. The current income gap between doctors and the United States and Japan and Singapore is very large.

China's current job performance wage system, the basic wage can only maintain the basic life of doctors, and performance pay is variable and variable pay, according to the hospital and the department's business income balance to a certain percentage of distribution to the departments, and 
then by the various departments in accordance with their own bonuses Accounting standards issued to the doctors. Reality The proportion of basic salary of TCM doctors is very low, the proportion of performance pay and bonuses is too large, or even $80 \%$. At the same time, the basic wage level of doctors in our country is affected by the title, working life and so on, and the professional and technical qualification of the re-scientific research, light practice, resulting in professional and technical qualifications can not fully reflect the clinical professional level. Performance evaluation indicators to health care workers and job responsibilities to complete the situation for the evaluation of the content, a single indicator, which concerned about the workload of medical staff will reduce the quality of medical care, but also does not reflect the knowledge, technology and other factors of production. So unreasonable salary structure, unscientific comprehensive evaluation system, too low basic salary plus drug addition charges, according to the project addition and other institutional arrangements, so that doctors pay more attention to economic indicators, ignoring the quality of work and social welfare indicators, and even through the improper channels to obtain gray income, increased the doctor-patient contradictions, affecting the doctor's social image.

The current hospital is facing many new challenges with and retains a number of sophisticated talents, is the hospital in the medical reform process and the healthy growth of the market to ensure that the fundamental guarantee. Medical professionals do not only pursue economic benefits that can satisfy their rich lives, but also pursue the social needs that can meet their personal values, the need for honor, and the need for spiritual enjoyment. And pay attention to the remuneration system of China's Hospital union is more of the external remuneration, the lack of attention to the internal remuneration of non-monetary, lack of non-monetary compensation system for studying abroad, business insurance, condolences and paid leave, the incentive role of professionals is not obvious.

\section{The Reform Direction of China's Medical Staff Compensation System}

Increase the government financial investment, the establishment of a solid higher salary of the investment security system, to expand infrastructure, large-scale experimental equipment and other inputs, dynamic adjustment of total wages, but to highlight the public hospital public welfare, the health of output. The establishment of financial compensation mechanism, subsidies for public health events to subsidize, improve the price of medical services, reduce the price of drugs, medical equipment, to determine the various forms of pay payment, such as by disease management, pay, disease diagnosis Grouping, etc. to determine the basic wage, the drug bonus income into medical service fees.

China's Hospital union salary system should dilute the performance of wages, the establishment of a higher level of compensation system to post, title, year for the evaluation of the fixed wage wage to reach more than $60 \%$ of total wages, supplemented by overtime allowance, Long - term incentive effect of the pay system. This is also a high level of medical and health performance of the developed countries Hospital union salary system arrangements common point, that is, "high salaries." In accordance with international practice and the professional characteristics of doctors, Hospital union doctors should pay the average salary level of 2.5 times the average wage, that is, about 10 million annual income. At the same time, it is necessary to establish a regulatory body to strengthen the supervision of the behavior of hospitals and doctors, highlight the characteristics of hospital public welfare, enhance the image of the social society of doctors.

The determination of salary performance indicators should be clear, scientific, controllable, operational and evidence-based. The evaluation of indicators should focus on the quality of medical services, the workload and cost control, and reflect the fairness, fairness and transparency of pay. The international compensation level is usually determined by the factor assessment method, such as the United States mainly in knowledge and skills, work environment, work complexity and other 10 factors to assess the doctor's salary level, according to the nature of each element and different circumstances, given different degrees of weight, Skills, the highest weight, the doctor's satisfaction with the income level is also higher. Therefore, China's Hospital union can be universal factor assessment method.

Improve the internal salary system of medical staff. At present, China's doctors a higher level of 
knowledge, more is the pursuit of deep-seated, invisible non-monetary remuneration. First, to provide high-level talent appreciation space and to better stimulates the medical staff's subjective initiative. Second, to provide a good working environment, such as the provision of laboratory equipment and the venue, gives medical personnel in the spare time to do research. Third, to create a good hospital culture, the establishment of a loose, allowing the experimental failure of the working environment, inclusive of high-level talent, so that they quickly into the hospital culture, to encourage high-level talent to carry out technological innovation. Fourth, according to the individual differences of medical personnel, to achieve differentiation incentives, through communication with different levels of medical personnel, targeted to develop a different pay distribution system to meet the diverse needs of different medical personnel, play the largest distribution of pay Incentive effect.

\section{Conclusion}

The design of the hospital salary system is a systematic and complicated project, which requires both scientific theory and method application. It also needs to have a good hospital culture and values. It is more important that the design of the whole salary system should be standing on the height of the hospital to achieve the strategic objectives. Whether the hospital's strategic pay system can play a role depends on the scientific and effective management of the pay system, the value orientation of the employee's pay and its integration with the hospital management environment. Therefore, the establishment of a scientific and effective hospital management system is an important guarantee to achieve the goal of salary reform, the hospital should be in the management structure, staffing, job placement, performance evaluation and so on to make a more comprehensive planning and set before implementation.

\section{Acknowledgements}

Fund Project: Sichuan hospital management and development research center project, Hospital union, under the background of the salary system reform research

Project Number: SCYG2017-28.

\section{References}

[1] Chang Qi, Yu Runji. Depth to help county hospital [J]. Chinese Hospital Dean, 2011 (08)

[2] Shao Rong, Zhang Ziwei. Comparative Analysis of the Causes of Formation of Hospital Groups in China and China [J]. Chinese Pharmacy, 2010 (41)

[3] Li Chen. How to strengthen the financial management and control system of hospital groups [J]. China Hospital Dean, 2009 (Z1)

[4] Sun Xun, Ou Chongyang. China's hospital group formation motivation analysis [J]. People's Liberation Army Hospital Management Journal, 2008 (11)

[5] Liu Yue, Zhang Shengquan. On the hospital group sustainable development path selection strategy [J]. Modern Hospital Management, 2008 (01)

[6] Xu Weifeng. Improve the internal control of hospital groups to improve the level of financial management [J]. Modern Hospital, 2008 (07) 